Sociologie et sociétés

\title{
Universalisation et traditionalisation de la discipline sociologique
}

Le cas du Québec francophone

\section{Universalization and Traditionalization of Sociology as a Discipline}

\section{The Case of French-speaking Quebec}

\section{Jean-Philippe Warren}

Volume 37, numéro 2, automne 2005

Le Québec et l'internationalisation des sciences sociales

Quebec and the Internationalization of the Social Sciences

URI : https://id.erudit.org/iderudit/012913ar

DOI : https://doi.org/10.7202/012913ar

Aller au sommaire du numéro

\section{Éditeur(s)}

Les Presses de l'Université de Montréal

\section{ISSN}

\section{8-030X (imprimé)}

1492-1375 (numérique)

\section{Découvrir la revue}

\section{Citer cet article}

Warren, J.-P. (2005). Universalisation et traditionalisation de la discipline sociologique : le cas du Québec francophone. Sociologie et sociétés, 37(2), 65-89. https://doi.org/10.7202/012913ar

\section{Résumé de l'article}

Cet article s'intéresse à la fondation historique d'un champ disciplinaire national, c'est-à-dire au processus de traditionalisation scientifique. Plus spécifiquement, il cherche à mieux comprendre, et ce en la comparant sans cesse au développement de la sociologie du Canada anglophone, comment la sociologie du Québec francophone a progressivement créé un espace référentiel, méthodologique, théorique et politique, et comment celle-ci a cherché, surtout dans les années 1960 et 1970, à promouvoir une connaissance enracinée dans la réalité nationale. C'est ainsi que l'auteur est amené à souligner trois dimensions inhérentes à la constitution d'un champ sociologique national, soit la définition d'une société globale, l'écriture d'une histoire, ainsi que la formulation de méthodes et de théories spécifiques marginalisant donc les dimensions idéologiques et institutionnelles invoquées par Harry H. Hiller dans ses travaux sur l'établissement des traditions sociologiques canadiennes. 


\title{
Universalisation et traditionalisation de la discipline sociologique
}

Le cas du Québec francophone'

\author{
JEAN-PHILIPPE WARREN \\ Département de sociologie et d'anthropologie \\ Université Concordia \\ 1455, boul. de Maisonneuve Ouest \\ Montréal (Québec), Canada $\mathrm{H}_{3} \mathrm{G}_{1} \mathrm{M} 8$ \\ Courriel: Jphwarren@aol.com
}

\begin{abstract}
Cet article s'intéresse à la fondation historique d'un champ disciplinaire national, à savoir au processus de traditionalisation scientifique en sciences sociales. Plus spécifiquement, il cherche à mieux comprendre, en la comparant sans cesse au développement de la sociologie du Canada anglophone, comment la sociologie du Québec francophone a progressivement créé un espace référentiel — paradigmatique et idéologique - et comment cette sociologie a cherché, surtout dans les années 1960 et 1970, à promouvoir une connaissance enracinée dans la réalité nationale. Ces dernières années, cette question a amené plusieurs chercheurs à s'interroger, dans une perspective de sociologie de la connaissance ou simplement dans une perspective historique et critique, sur le rapport épistémologique, théorique et politique que la sociologie entretient nécessairement avec la société concrète (Crawford, Shinn et Sörlin, 1993; Trindade, 2005; McLaughlin, 2005). «Historiquement, peut-on lire dans un rapport de l'Unesco sur "Les sciences sociales dans le monde", les sciences sociales se sont développées nationalement. La recherche s'est surtout concentrée sur les questions nationales» (Kazancigil et Makison, 2001, p. 9). Cette affirmation semble cependant de moins en moins
\end{abstract}

1. Je tiens à remercier les deux évaluateurs anonymes de la revue, ainsi que Yves Gingras, pour leurs judicieux commentaires sur une version préléminaire de ce texte. 
acceptable dans le contexte d'une croissance des échanges internationaux et d'une délocalisation de la recherche. Notre objectif est donc de saisir la constitution progressive, au Québec francophone, d'un champ sociologique national afin d'établir, dans un deuxième temps, les dimensions constitutives de tout champ national.

Nous appelons « universalisation» (et, inversement, «traditionalisation») l'accroissement de la reconnaissance d'un paradigme scientifique et «internationalisation» (et, inversement, «nationalisation») le mouvement concret de production, diffusion et consécration du savoir scientifique. Ainsi, une science est universalisée dans ses méthodes et ses théories, tandis qu'elle est internationalisée dans ses conditions institutionnelles et organisationnelles (soutiens financiers, cadres institutionnels, réseaux d'échange, instances de vulgarisation, etc.). En d'autres termes, nous désignons ici par processus de traditionalisation la constitution historique d'une tradition disciplinaire (Nisbet, 1984). La condition concrète d'apparition d'une tradition scientifique est la constitution d'un champ scientifique national (Genov, 1989a). On reconnaît d'ordinaire que l'internationalisation de l'activité scientifique se mesure à l'aune de la circulation des personnes, des textes et des objets, le mode de production du savoir et le financement de la recherche (Crane, 1972; Gingras, 2002). À noter, comme il faudra y faire sans cesse attention dans la suite du texte, que l'universalisation et l'internationalisation sont des processus relativement disjoints, dans la mesure où l'on peut imaginer des conditions de production locale des connaissances dans le contexte d'une science dont la tradition déborde le cadre de la nation (le cas est fréquent, pour dire le moins, en ce qui concerne les sciences dites exactes) — et inversement.

Nous ne pouvons pas plus nier le caractère international de la pratique sociologique que le repliement de celle-ci sur les frontières nationales, ethniques, de classes ou de genres. «On croit souvent que la vie intellectuelle est spontanément internationale. Rien n'est plus faux. La vie intellectuelle est le lieu, comme tous les autres espaces sociaux, de nationalismes et d'impérialismes [...]» (Bourdieu, 2002b; 1992; 2000). C'est à partir de cette prémisse que cet article s'intéresse à la constitution d'un champ national dans le contexte de la société québécoise. Les œuvres sociologiques circulent entre le Québec et les autres provinces ou pays, certes, seulement elles circulent parfois difficilement, comme si une résistance continuelle s'exerçait dans le sens contraire de l'universalisation (ou de l'internationalisation) de la discipline. Situé historiquement au carrefour de quatre empires, à savoir, dit grossièrement, un empire politique (Angleterre), un empire économique (États-Unis), un empire culturel (France) et un empire religieux (Église catholique), le Québec francophone est un laboratoire particulièrement intéressant pour dégager les mécanismes de cette résistance épistémique ou politique et les structures institutionnelles ou sociales qui sous-tendent cette tension ${ }^{2}$.

2. Sur la question de l'autonomie du champ québécois par rapport aux autres disciplines, qui n'est pas notre perspective ici, lire, entre autres, Fournier, Germain, Lamarche et Maheu (1975). Adoptant une lecture qui se situe en général au niveau de l'évolution départementale, Marcel Fournier a écrit plusieurs articles qui s'attachent à décrire la progressive délimitation de la sociologie par rapport aux autres savoirs académiques (théologie, philosophie, droit, journalisme, etc.). On trouvera une illustration de ses thèses dans L'Entrée dans la modernité (1986). 
Il permet de mieux comprendre comment les clivages culturels et les aspirations à établir une connaissance objective tout à la fois se conjuguent et s'opposent de manière particulière dans l'histoire de la pratique sociologique. Société de culture fragile et incertaine, et, pour cette raison, prompte à succomber, comme ailleurs en de semblables contextes, à des crispations identitaires ou à des mouvements nationalistes, tout autant qu'à s'ouvrir, en raison de sa périphérie, aux grands courants qui traversent les champs plus centraux ou métropolitains, le Québec francophone (ou encore, avant 1960, le Canada français) permet d'illustrer quelques-uns des principes primordiaux qui orientent l'institutionnalisation d'un champ national.

\section{LE CHAMP DE LA SOCIOLOGIE QUÉBÉCOISE FRANCOPHONE DANS L'ÉTAT-NATION CANADIEN}

Commençons par le plus banal. Étant posé l'existence d'un champ sociologique national $^{3}$, les sociologues qui œuvrent à l'intérieur de ce champ auront tendance à ignorer les collègues contre qui ce champ se définit. En d'autres termes, la sociologie, réceptive à l'influence de plusieurs courants de pensée étrangers, demeurera plus ou moins fermée à d'autres courants qui menacent, ne serait-ce que par le simple fait du dialogue, l'autonomie de la pratique nationale, ou qui plus simplement ont besoin, pour être assimilés, d'être médiatisés ou traduits - comme dans le cas, par exemple, des praticiens français des sciences sociales qui collaborent davantage, pour des raisons évidentes d'histoire et de langue, avec le Canada et la Belgique que les praticiens français des sciences, dont le champ est plus décontextualisé et délocalisé.

Étant donné son caractère binational, il est tentant d'étudier l'autonomie disciplinaire à l'intérieur même des cadres de la fédération canadienne, et ce d'autant plus que le Canada français s'est longtemps défini par son opposition au Canada anglais au point, paradoxalement, de l'oblitérer de sa conscience collective. Ce dialogue brisé, voire impossible, entre les deux solitudes, est à l'image des échanges entre les sociologues canadiens-anglais et canadiens-français tout au long du Xx siècle (Brooks et Gagnon, 1994; Dion, 1991; Morris, 1993; Salée, 1999; Schwartz, 1991; Warren et Nielsen, 2003). Alors même qu'ils font face à des défis sociaux ou économiques semblables et qu'ils empruntent à des perspectives paradigmatiques parentes, ils demeurent incapables de surmonter l'invisible frontière qui parfois, au cœur d'une même ville, les sépare en deux blocs hermétiques (Warren, 2003b).

Les années 1950 ont sans doute correspondu, dans l'histoire de la discipline, à un des plus forts moments du sentiment d'un progrès de la science sociologique vers l'établissement d'une connaissance universelle. La raison allait triompher des arbitraires

3. Pour une définition du champ tirée de l'œuvre de Pierre Bourdieu, lire, entre autres, l'article de 1976 dans lequel le champ «comme système des relations objectives entre les positions acquises (par les luttes antérieures)» est défini comme «le lieu (c'est-à-dire l'espace de jeu) d'une lutte de concurrence qui a pour enjeu spécifique le monopole de l'autorité scientifique inséparablement définie comme capacité technique et comme pouvoir social» (Bourdieu, 1976, p. 91; 2002b). Pour un exemple particulièrement éclairant d'autonomisation du champ scientifique sur la base de sa professionnalisation et de sa formalisation, lire Gingras (2003). 
historiques. Mais puisque cette croyance s'appuyait sur le déni du caractère national de la pratique sociologique, il allait s'ensuivre maints malentendus, comme lorsque la science états-unienne de l'après-guerre voulut se faire passer, à la faveur du triomphalisme des valeurs libérales américaines et de la croissance spectaculaire du complexe techno-industriel, pour la seule science universelle. C'est C. B. Macpherson qui déclarait en 1957:

It can no longer be said that there are two sociologies, as there are two histories, in Canada. The sociologists of both languages are working with the same concepts and using the same techniques of analysis, derived in large measure from American sociology. And since the French-Canadian sociologists have broken away from obsessive interest in cultural uniqueness, the lines of convergence between French- and English-Canadian sociology are plain. (Macpherson, 1957, p. 219)

Notons dans cette déclaration deux suppositions qui semblent, pour l'auteur, aller de soi: le progrès de la science sociale se fonde d'abord sur les concepts et la méthodologie développés par les sociologues américains; l'obsession nationalitaire est celui de la société périphérique et ne saurait en aucun cas être celui de la métropole ${ }^{4}$.

L'optimisme de Macpherson allait faire long feu. Bien que l'on puisse noter une parenthèse, qui dure à peu près dix ans (1950-1960), pendant laquelle une partie de la sociologie canadienne-française cherche à se fédérer au champ scientifique canadienanglais (Jean-Charles Falardeau est élu président de la Canadian Political Science Association/Association canadienne de science politique ${ }^{5}$, et quelques praticiens francoquébécois des sciences sociales s'inscrivent aux sessions annuelles de cette association ou publient des articles dans sa revue), cet interlude s'explique d'abord par une scission au sein du champ nationaliste québécois, les fédéralistes canadiens-français tentant de démontrer, dans l'exercice de leur pratique elle-même, la possibilité de fonder une référence collective canadienne bilingue et biculturelle ${ }^{6}$.

Il ne faudrait pourtant pas exagérer la collaboration véritable entre les sociologues du Québec francophone et ceux du ROC (Rest of Canada). D'une part, ce rapprochement a été permis par la déclaration de la Seconde Guerre mondiale, guerre qui a forcé les étudiants québécois à se diriger vers les universités américaines, eux dont le tempérament et les intérêts les destinaient à compléter leur formation en Belgique ou en France. Sitôt la guerre terminée, le mouvement spontané vers l'Europe a repris, et, au tournant des années 1970, les diplômés des universités américaines ne représentaient

4. Everett C. Hughes, un auteur pourtant allergique aux témoignages d'aveuglement ethnocentrique, n'en déclarait pas moins, dans une lettre au père Georges-Henri Lévesque datée de 1977: «The people who are called minorities are really the most interesting. They have to adapt themselves to the rest of us» (Hughes, 1977).

5. Falardeau fut aussi membre, pendant six ans, et président de 1950 à 1952, du Conseil canadien de recherche en sciences sociales (Canadian Social Science Research Council) et conseiller (1948-1950) de l'exécutif de la Société canadienne d'histoire (Canadian Historical Association).

6. Pierre Elliott Trudeau faisait de l'usage de la raison l'article premier de sa politique fonctionnelle, dans la même mesure où Falardeau faisait de la raison, couplée avec le dialogue et le respect des différences, la base première du développement des sciences sociales. 
qu'une minorité du corps professoral franco-québécois dans les départements de sociologie (Warren, 2005). D’autre part, en dehors de la figure emblématique de la sociologie franco-québécoise des années d'après-guerre, Jean-Charles Falardeau ${ }^{7}$, rares sont les praticiens de l'un et l'autre groupes linguistiques qui se lient à travers des initiatives réelles. Maurice Lamontagne a sans doute discuté et échangé intensivement avec ses collègues des autres provinces, cependant on cherche en vain dans son œuvre une collaboration académique concrète. Publier des articles dans des revues scientifiques canadiennes-anglaises quand des équivalents canadiens-français n'existaient pas encore (Recherches sociographiques sera fondée en 1960, et Sociologie et sociétés en 1969) ou encore des chapitres dans des collectifs bilingues ${ }^{8}$, voilà en quoi consiste l'essentiel de l'interpénétration des deux communautés linguistiques en ces années. Enfin, il faut insister sur le fait que le dialogue scientifique et intellectuel entre les sociologues du Québec francophone et ceux du ROC s'est établi par l'intermédiaire de la sociologie américaine, plus spécifiquement de l'École de Chicago. C'est avant tout par le détour des États-Unis, où les uns et les autres avaient été formés, que les praticiens de l'une et l'autres langue ont pu se considérer héritiers d'une même tradition de pensée. Il est à cet égard révélateur que Everett C. Hughes, un Américain, ait eu des contacts profonds et suivis avec les sociologues de l'Université Laval, qu'il ait été invité à enseigner à cette institution ${ }^{9}$ et ait consacré une monographie (French Canada in Transition) aux transformations sociales qui affectaient le groupe canadien de langue française, tâchant de cerner les tensions qui surgissaient de l'intégration d'une minorité ethnique dans le système industriel nord-américain, alors que Carl A. Dawson, son collègue à l'Université McGill jusqu'au départ de Hughes en 1938, un Canadien ayant grandi en NouvelleÉcosse, saisissait l'occasion fournie par la collection dirigée par Mackintosh sur les aires de colonisation pour analyser, tout à l'opposé, le peuplement de l'Ouest canadien, sans se soucier de faire un effort pour connaître ses confrères sociologues de Québec ou Montréal (Shore, 1987). Dans les années 1950, au moment, donc, du dialogue le plus fécond entre sociologues canadiens anglophones et francophones, l'entente se fait sur

7. Indice de cette collaboration, Jean-Charles Falardeau signe avec Frank E. Jones, professeur à l’Université MacMaster, «La sociologie au Canada» (1956).

8. Ainsi, l'ouvrage bilingue de l'historien américain Mason Wade (1960). Aussi, l'édition par Falardeau d'un colloque tenu à Québec dans les deux langues, «Essais sur le Québec contemporain/Essays on Contemporary Quebec» (1953). À noter que la revue du Département de relations industrielles de l'Université Laval, le Bulletin des relations industrielles, est bilingue. Quant à la revue Culture publiée par l'École de service social, elle se permet, comme quelques autres, de publier à l'occasion des articles en anglais. Quand les colloques sont organisés à l'extérieur de la province de Québec, il semble que l'usage de l'anglais soit de mise, comme en témoigne l'organisation de la Conference on Research in Industrial Relations, tenue à Toronto en 1947, où les pères Gérard Dion et Émile Bouvier firent leur présentation dans la langue de Shakespeare. La volonté d'attribuer un doctorat honoris causa à Harold A. Innis par l'Université Laval en 1946 se voulait le signe de la solidarité de la vie académique canadienne. Finalement, les échanges de professeurs entre universités à la fin de la guerre, par exemple entre l'Université Laval et l'Université de Toronto (Falardeau y enseignera quatre semaines en 1948), permettaient, croyait-il, de favoriser plus concrètement les échanges intellectuels et savants.

9. En 1942-1943. Il écrit aussi un Programme de recherche sociale pour le Québec à l'intention des professeurs de l'École des sciences sociales de l'Université Laval. 
la base d'un rejet commun de la tradition britannique ou française. Le champ national n'est pas aboli; il change seulement de visage.

Guy Rocher, disciple de Talcott Parsons et diplômé de l'Université Harvard, a noté que, dès les années 1960, la petite fenêtre ouverte sur le ROC se referme, ce que démontre, entre autres signes, la création en 1969 d'une association indépendante, l'Association canadienne des sociologues et anthropologues de langue française. Dans les congrès annuels de la Canadian Political Science Association (tout autant que dans ceux de la Canadian Sociology and Anthropology Association/Société canadienne de sociologie et d'anthropologie [association bilingue], fondée en 1964), le nombre de communications présentées par des francophones atteint progressivement une proportion insignifiante de l'ensemble. Au congrès de 1967, par exemple, congrès qui se tenait, précisons-le, à l'Université Carleton à Ottawa, une seule personne sur près de 90 participants était francophone. Au congrès de 1972, tenu à l'Université McGill à Montréal, moins de $5 \%$ des communications étaient présentées par des Québécois francophones (Rocher, 1990). Aujourd'hui, à peine plus de $5 \%$ des articles publiés dans les deux grandes revues canadiennes de sociologie le sont par des auteurs francophones, et la moitié de ces articles ont été traduits préalablement en anglais (Nielsen et Warren, 2003).

Au Canada, cette clôture de la sociologie franco-québécoise apparaît d'autant plus surprenante que les étapes de son développement ressemblent fort à celles de la sociologie du ROC. Dans les années 1960, l'engouement pour la pensée de la décolonisation inspire les travaux des universitaires d'un bord et de l'autre de la rivière Outaouais. Dans les années 1970, le marxisme gagne en popularité chez les anglophones et les francophones. Dix ans plus tard, le développement des groupes de recherche et la course à la productivité nourrissent d'un océan à l'autre la volonté de créer des réseaux nationaux et internationaux. Récemment, la thématique de la mondialisation, accompagnée d'un discours autour de l'abolition des frontières et du flux sans contraintes des marchandises et des idées, conduit les sociologues à renoncer à la défense de leurs frontières académiques et intellectuelles ou, à l'opposé, à s'unir autour du projet de l'altermondialisation. Chaque fois, pourtant, la question nationale finit au Québec par orienter les débats vers des voies parallèles mais non convergentes. Pendant les années 1960 et 1970, c'est-à-dire pendant la période de nation-building, par exemple, la sociologie canadienne-anglaise cherche à s'annexer une sociologie canadienne-française qui résiste à ses invitations. Se définissant alors par opposition à la sociologie américaine, la sociologie canadienne de langue anglaise avait peu à craindre d'une étroite association avec la sociologie franco-québécoise. La difficulté pour elle, c'était que les théories de la dépendance, dont André Gunder Frank par exemple s'était fait le spécialiste, théories qu'elle appliquait à la réalité nationale pour démontrer la nécessité de prendre ses distances par rapport au géant américain, étaient recyclées dans l'argumentaire des sociologues canadiens-français pour justifier leur lutte en faveur de la décolonisation économique, sociale, politique et culturelle des Québécois francophones. Quant au marxisme franco-québécois, s'il affiche une originalité, c'est par son incessant débat 
avec la question nationale. Rares sont les articles, rarissimes sont les livres publiés par les marxistes des années 1970 qui ne ménagent pas au moins une section à l'exploration des rapports entre nations et classes sociales.

C’est dans les années 1960 que s'est révélée avec force la volonté de fonder une tradition sociologique nationale spécifiquement canadienne. Non pas que, auparavant, une telle volonté n'existait pas en puissance; mais il lui manquait, pour s'exprimer avec vigueur, des institutions relativement importantes ${ }^{10}$; de manière plus fondamentale encore, il lui manquait d'être nourri par un véritable mouvement de nation building canadien. On dira que, pour le Canada français, il existait une référence collective depuis au moins le XIX ${ }^{\mathrm{e}}$ siècle (Dumont, 1993), et l'on aura raison; seulement, cette référence était doublement ambiguë: la société canadienne-française était une société tronquée (elle englobait seulement cette portion du Canada qui parlait français) et elle était instituée pratiquement et moralement par l'Église catholique (ce qui ajoutait à la première nationalité des Canadiens français une «seconde», puisque leur «pays» était aussi la catholicité [Gagné et Warren, 2003]). Quant à la référence collective proprement canadienne-anglaise, elle prend forme au moment de la défaite de l'ancienne référence impériale (George Grant publiant son Lament for a Nation l'année même de l'élection de Pierre Elliott Trudeau comme député du Parti libéral fédéral) et s'exacerbe par la concurrence que lui mène un impérialisme américain affaibli mais toujours menaçant.

\section{TROIS DIMENSIONS SUFFISANTES D'UN CHAMP SOCIOLOGIQUE NATIONAL}

Ce bref parcours historique mettant en parallèle les sociologies canadiennes anglophone et francophone a permis de dégager à la fois les convergences (pratiques, théoriques et idéologiques) et l'isolement de deux traditions instituées dans le cadre officiel d'un même État-nation. Comme nous l'annoncions en introduction, la seconde partie de cet article interroge, sur un mode plus formel, les dimensions premières et suffisantes de reconnaissance d'un champ sociologique national. Il s'agit de comprendre comment la formation d'un champ national est informée par une dynamique qui fait se rejoindre l'horizon d'une société globale, l'écriture d'une histoire et la formulation de méthodes et de théories spécifiques. Les quelques éléments esquissés dans la première partie serviront ainsi de matériaux pour reconstituer, par hypothèse, le processus général de traditionalisation de la discipline sociologique.

\section{L'horizon d'une société globale}

D'abord, la traditionalisation est liée à la situation de la recherche dans l'horizon d'une société globale (Fournier, 2001 et 2002). En ce qui concerne la nation québécoise, on

10. Les chiffres sont éloquents. En 1963, il y avait 106 sociologues enseignant dans les universités canadiennes; en 1969, ils étaient 548 et, en 1981, 1427 (Hofley, 1992, p. 105). De tels chiffres n'expliquent à l'évidence pas tout, mais ils permettent de mieux comprendre dans quel contexte d'effervescence s'est posée la question de l'autonomie du champ scientifique dans les années 1970. 
peut faire remonter cette étape à 1953, au moment de la publication des Essais sur le Québec contemporain, dans la préface desquels Jean-Charles Falardeau faisait refluer le Canada français sur les frontières de la province; mais c'est véritablement en 1960, avec le début de la Révolution tranquille et l'élaboration d'un programme indépendantiste (RIN, Parti pris, etc.), que le Québec devient, dans la perspective que les sociologues héritent de la politique en même temps qu'ils l'informent, une société globale. En 1962, par exemple, l'emploi par un Fernand Dumont de l'expression ambiguë «société globale canadienne-française» démontre que la transition n'est pas encore consommée (Dumont, 1962). En 1970, de telles hésitations ne sont plus de mises: «C’est ici, écrit Marcel Rioux, que naît la question du Québec. Il n’est plus question du Canada français, ni de bilinguisme, ni de biculturalisme, mais des pouvoirs de l'État du Québec et de l'organisation de la vie collective des Québécois» (Rioux, 1969, p. 151). Cette déclaration est à la fois piquante et tragique, dans la mesure où c'était alors par un pur artifice qu'on arrivait à faire s'égaler Québec et société globale nationale: en abandonnant les Canadiens français hors Québec (lesquels continuèrent à s'appeler Canadiens français, malgré des velléités émergentes de recomposer leur référence collective sur la base de l'appartenance provinciale) et en oblitérant l'existence des Québécois anglophones, quitte, de l'heure seulement où l'on se rappelait leur présence, à les expulser pour ainsi dire symboliquement de la référence collective nouvelle en les rebaptisant Canadiens ou, mieux, Canadians.

En soulignant cette dimension d'horizon de la société globale, nous n'entendons pas une recherche axée uniquement ou prioritairement sur l'analyse des cadres les plus généraux du social, mais d'abord la volonté de replacer les avancées de la recherche dans le contexte d'une société en formation. L'étude des objets concrets semble avoir pour fin, explicite ou implicite, avouée ou spontanée, une meilleure compréhension (dans le sens double d'un éclaircissement et d'une saisie cognitive) du «monde commun» dans lequel s'inscrivent les sociologues.

The danger for Canadian sociology, écrivait un auteur en 1970 dans un collectif intitulé L'avenir de la sociologie au Canada, is that wholesale application of U. S. findings or theory due to a paucity of Canadian data may be highly misleading in the context of Canadian society. We should beware of equating what is American with what is universal merely because of the present predominant position of American sociologists. (Coburn, 1970, p. 39)

C'est ce genre de commentaires que Fernand Dumont reprendra quand il invitera ses collègues à se préoccuper davantage de la «société d'ici».

Il est évident que la langue joue un rôle important dans le processus de traditionalisation de la discipline, quoique celui-ci ne saurait être exagéré. Les sociologues canadiens-anglais ont cherché à autonomiser leur champ scientifique par rapport à celui des États-Unis dans les années 1960, alors que les sociologues québécois se sont servis des influences françaises pour asseoir la légitimité d'une tradition propre. Il n'empêche que le partage d'une langue favorise l'institution de traditions, nous ne dirons pas communes, mais certainement parentes, un peu comme la proximité géo- 
graphique contribue à resserrer certains liens. Ce qui a permis l'établissement d'une réflexion sociologique originale au Québec, c’est, en partie, cette double réalité linguistique et géographique qui situait spontanément la province aux confluents des influences états-uniennes, britanniques et françaises ${ }^{11}$.

Ce que nous devons souligner, c'est que la définition de la société globale tend à recouper celle de l'État-nation, avec pour conséquence que la tradition sociologique se trouve liée à celui-ci de manière profonde et non accessoire. Il peut, certes, exister une définition de la société globale qui transcende, pour la communauté de sociologues, les espaces des États-nations, ainsi que ce fut et demeure le cas pour l'Amérique latine (Garretôn, Murmis, de Sierra et Trindade, 2005), mais les espaces nationaux ne s'en trouvent pas effacés pour autant; qu'il suffise d'affirmer ici que, en ce qui concerne l'Amérique latine (mais cela vaut pour d'autres régions), des instances et institutions particulières, supranationales (ainsi le Programme international d'études avancées de la Maison des sciences de l'homme à Paris) ont pu jouer le rôle, pour un certain groupe de chercheurs, de structures étatiques par défaut — un rôle qui, sans être mineur, n'en est pas moins fragile. À rebours, l'existence de telles structures ne saurait être une condition suffisante si elles ne sont pas saisies dans l'horizon de la société globale nationale, ainsi que nous l'apprend le cas de la sociologie canadienne-anglaise, elle qui a été suscitée par le développement du providentialisme d'État dans les années 1950 et 1960, et qui a pourtant, par la proximité de langue, de culture et de géographie, grandi dans l'ombre de la sociologie américaine. La fragilité d'une sociologie canadienne put être ainsi adéquatement décrite comme "an ideational reflection of the arrested development of Canada's national sovereignty» (Stolzman et Gamberg, 1975, p. 99).

\section{Écriture d'une histoire}

La seconde dimension consiste à se donner une histoire. Cette écriture permet de fonder la légitimité d'un champ scientifique distinct en renvoyant son origine à une incertaine tradition de pensée. Il existe à cet égard deux perspectives, chacune ayant sa pertinence selon le contexte de traditionalisation de la discipline sociologique. D'une part, l'on peut, comme Jean-Charles Falardeau a tenté de le faire en commémorant les œuvres de Léon Gérin ou Étienne Parent, souligner la continuité historique entre les sociologues d'aujourd'hui et ceux du passé. Il s'agit moins d'harnacher la réflexion la plus contemporaine par le renvoi à quelque filiation hypothétique que de tâcher de montrer comment les questions autour desquelles tournent les débats académiques actuels prolongent celles posées par les premiers sociologues faisant face aux boule-

11. En 1997, 95 \% des professeurs de sociologie états-uniens avaient reçu leur formation doctorale aux États-Unis, alors que cela était le cas de $35 \%$ des professeurs canadiens (42\% ayant reçu leur formation au Canada et $11 \%$ en Angleterre) (McLaughlin, 2004, p. 89-91) - la proportion de doctorants américains étant en très légère baisse par rapport aux années 1970. Au Québec francophone, alors que les diplômés de France dominaient les départements de sociologie en 1970, la situation récente correspond bien, ici aussi, à une nationalisation (mais point pour autant à une traditionalisation) de la formation doctorale (Warren, 2005). 
versements suscités par les forces de l'industrialisation et de la démocratie. D'autre part, l'on peut, privilégiant la rupture à la continuité, insister sur le caractère soudain de l'apparition de la sociologie dans le paysage intellectuel. L'histoire de la discipline est décrite selon une opposition avant/après, opposition qui oblige presque nécessairement à une lecture téléologique et héroïque des commencements de la science sociale. Cette lecture, privilégiée par plusieurs historiens québécois de la discipline, a contribué à renforcer l'image d'une sombre grande noirceur et d'une lumineuse Révolution tranquille. À la période de la préhistoire de la discipline (confusion des jugements de faits et des jugements de valeurs, faible institutionnalisation, amateurisme, etc.) devait forcément répondre une période de l'âge d'or (scientificité, croissance phénoménale de l'enseignement supérieur, professionnalisme, etc.) (Fournier, 2000).

Il n'y a pas d'ordre chronologique dans la présentation qui vient d'être faite de l'historisation de la pratique sociologique. Les deux perspectives ici définies peuvent coexister de manière concurrente ou encore se succéder dans le désordre. On note ainsi une insistance plus grande sur la continuité que sur la rupture dans les années 1960 que dans les années 1980, alors qu'on semble revenir à une lecture moins dichotomique depuis quelques années. Ces changements trahissent tout autant une reformulation des débats à l'intérieur du champ sociologique qu'ils reflètent des bouleversements sociaux et politiques dans le reste de la société. Cependant, le résultat, souhaité ou simplement inévitable (par la seule vertu de faire de quelques sociologues habitant un territoire un groupe distinct ${ }^{12}$ ), de l'écriture d'un récit historique, c'est de circonscrire les dimensions de constitution d'un champ sociologique national. L'histoire n'est jamais celle de la dispersion de la discipline, mais toujours celle de sa professionnalisation et de son institutionnalisation, parce que le $\mathrm{xx}^{\mathrm{e}}$ siècle a correspondu, en Amérique du Nord surtout, mais aussi, dans une moindre mesure, partout ailleurs en Occident, à une redéfinition de la pratique sociologique sur une base départementale. Cette division des universités en départements, qui allait se révéler d'une solidité étonnante tout au long du dernier siècle (Abbott, 2001), n'est pas uniquement adaptée aux conditions nouvelles de la recherche et de l'embauche (les doctorats devenant des visas entre ces pays hermétiques qui sont devenus les disciplines), mais elle est aussi particulièrement favorable à la constitution d'un champ disciplinaire propre, ce que prouvent à la fois la création de revues et la fondation d'associations professionnelles qui vont permettre l'émergence d'une identité moins équivoque que par le passé.

Qu'on ne s'y méprenne, et il faut y insister, l'écriture d'une histoire disciplinaire sur un mode national a un effet autant rétrospectif que performatif. C'est sur la base d'une reconnaissance d'une certaine originalité «scientifique» que des sociologues éprouvent le besoin de mieux circonscrire le développement de la discipline; mais dans le

12. On peut avoir une idée de cet arbitraire en prenant pour exemples des cas litigieux de «nationalisation» de sociologues. Michel Freitag, né en Suisse et formé en France, appartient-il à la tradition québécoise du simple fait d'avoir été embauché à l'UQAM? Everett C. Hughes peut-il être considéré un sociologue québécois pour avoir travailler une dizaine d'années à l'Université McGill et un an à l'Université Laval, avant de s'en retourner faire carrière à l'Université de Chicago? 
même mouvement où ils cherchent à dresser la carte d'un «territoire sociologique», ils donnent au champ national, souvent volontairement, une force et une cohérence que celui-ci n'avait pas auparavant. La traditionalisation d'une discipline est ainsi un effet de l'histoire autant que de discours.

\section{Des méthodes et des théories spécifiques}

La troisième dimension consiste à s'attribuer des méthodes et des théories différentes de celles employées par les champs nationaux avoisinants ou ceux avec lesquels le groupe national est en concurrence la plus directe (Southcott, 1994). Au Québec, Fernand Dumont a sans doute été celui ayant le plus plaidé pour le développement d'une pensée sociologique qui reflète les réalités sociales de la francophonie nordaméricaine (Dumont, 1976). Par exemple, il dénonçait le marxisme des années 1970 en tant que produit d'importation sans lien véritable avec la situation objective du peuple québécois, le marxisme étant, selon son dire, un vêtement idéologique taillé pour un autre siècle et un autre continent. Depuis les travaux de Dumont, le Québec francophone n'a eu, outre peut-être Marcel Rioux, aucun méthodologue ni théoricien d'envergure qui ait cherché à fonder école sur la base d'une reconnaissance du caractère distinct de la réalité québécoise. Cela permet d'expliquer pourquoi le champ sociologique national québécois demeure, par rapport aux États-Unis, pauvrement traditionalisé (sans être pour cela moins institué): il est en vérité sans théorie autochtone comme sans méthode - une situation qui est, après tout, propre à toute région ou pays de petite et moyenne tailles scientifiques (Gingras, 2002, p. 35). Rares sont les cours spécifiques offerts aux étudiants des universités de la province dans lesquels ils pourraient se familiariser avec les grands noms québécois de la discipline; leurs auteurs de référence s'appellent Max Weber, Émile Durkheim et Talcott Parsons, ce qui va de soi quand on ne peut tirer une perspective articulée et structurée de la lecture du Lieu de l'homme ou une démarche de recherche de Le besoin et le désir — notons que, en revanche, l'école formée autour des travaux du Groupe interuniversitaire de recherche sur la postmodernité, dirigé par Michel Freitag, très structurée théoriquement, a peu abordé jusqu'à présent la question du Québec dans les pages de la revue Société.

C’est ici que le caractère autoréférentiel du champ national apparaît le plus clairement, une référentialité dont on se fera une idée approximative à partir des mesures de bibliométrie. Encore une fois, outre les théories et les méthodes, qui sont censées fonder l'originalité d'une tradition, on constatera sans surprise que ce sont souvent d'abord les objets de recherche - localisés, singuliers, contextualisés - qui donnent au champ national sa force et sa cohérence. Ce phénomène découle de trois causes: d'abord, de la particularité des objets eux-mêmes (les ghettos noirs américains n'existent pas dans d'autres régions du monde), de leur pertinence sociale ensuite (les études sur l'intégration linguistique des immigrants attirent davantage l'attention des chercheurs en milieu minoritaire) et, enfin, de leur traductibilité (les concepts de middle class, de bourgeoisie et de Bürgertum, dont on postule la similarité, désignent en fait des caté- 
gories distinctes et supposent conséquemment, jusqu'à un certain point, des mesures empiriques légèrement différentes).

\section{QUELQUES CONSIDÉRATIONS GÉNÉRALES}

Il n'est pas évident de juger dans quelle mesure le processus de traditionalisation de la discipline a été heureux ou, au contraire, source d'un handicap pour le Québec francophone. D'un côté, fermée sur elle-même, dans la mesure où elle demeurait tournée vers l'analyse de la société globale québécoise et tentait de s'inscrire dans une histoire propre, on pourra se réjouir qu'elle a toujours dû s'ouvrir aux courants de pensée qui traversaient le champ scientifique en Europe ou aux États-Unis ${ }^{13}$. Plusieurs grands sociologues franco-québécois ont été davantage des représentants d'une école particulière (ainsi Guy Rocher pour Talcott Parsons, etc.) que des fondateurs (à l'exception, encore une fois, de Fernand Dumont et Michel Freitag). D'un autre côté, on pourrait tout aussi bien prétendre que ce phénomène d'ouverture en est véritablement un de dépendance, les pays économiquement et culturellement marginalisés ayant une proportion plus élevée de références extranationales, et opposer ainsi à la critique de l'autarcie des uns la nécessaire défense de l'autonomie de la production scientifique. L'étude de ce débat entre nationalistes et internationalistes serait en soi stérile s'il ne révélait, par un autre biais, la tension irréductible entre nationalisation et internationalisation dont la sociologie est, comme nous l'avons souligné plus haut, de par sa nature propre, spontanément l'objet.

La croyance en l'universalité de la science sociologique n'est pas, assez curieusement, incompatible avec la création de sociologies nationales, et ce pour la bonne raison que cette universalité est factice. La pratique sociologique ne saurait atteindre le degré de formalisation et d'abstraction des sciences dites exactes, étant toujours portée par les idéologies en vogue et, plus généralement, par le contexte sociohistorique (Dumont, 1974). C'est ainsi que les sciences exactes franco-québécoises se sont assez rapidement fondues dans le mouvement scientifique américain et se sont branchées sur le système universitaire de Boston ou New York, alors que les sciences sociales ont continué longtemps, et encore aujourd'hui, à s'alimenter aux courants de pensée européens, et d'abord aux courants de pensée français ${ }^{14}$. Léon Gérin représente un bel exemple d'un savant ayant œuvré à la constitution d'un champ sociologique national, alors même qu'il travaillait, selon son dire, à la diffusion d'une science universelle. D'une part, parce que ce dernier retraduisait dans le langage des craintes et des espoirs du Canada français de la fin du XIX ${ }^{\mathrm{e}}$ siècle les enseignements de Demolins et de

13. Cette affirmation, vraie pour la sociologie, l'est a fortiori pour les sciences dites exactes. Lire, pour les années 1970, S. S. Blume et M. J. Chartier, "The Effect of Biculturalism on Science in Canada», Institut d'histoire et de sociopolitique des sciences, Université de Montréal, septembre 1974; cité par Fournier, Germain, Lamarche et Maheu (1975, p. 129).

14. Les sciences sociales ont constamment un plus faible taux de collaboration internationale que les sciences pures, comme la physique, et même que les sciences plus appliquées, comme l'ingénierie. L'internationalisation est ici proportionnelle, entre autres, au degré de formalisation des disciplines, elle-même liée au caractère abstrait et générique de l'objet (atome, corps humain, rapport social, etc.) (Gingras, 2000). 
Tourville, Gérin ayant trouvé dans la science sociale de l'école le playsienne de la branche tourvillienne la possibilité de réconcilier son désir d'engagement avec sa volonté de faire œuvre de science. D'autre part, et de manière tout aussi importante, parce que, du fait de son isolement, Gérin ne pouvait guère apporter des modifications à la méthode élaborée par l'abbé Henri de Tourville sans susciter une certaine méfiance de la part des tenants de l'orthodoxie (eux qui, paradoxalement, avaient consommé une scission avec l'école de la réforme sociale). C'est ainsi qu'après avoir entretenu une longue et assidue correspondance avec ses maîtres parisiens, Gérin a dû poursuivre à peu près seul son patient travail d'affinement de la nomenclature tourvillienne (Warren, 2003a). Sous le reproche de gauchir le modèle d'investigation monographique formulé par l'auguste sociologue, il ne put publier la suite de son travail, pourtant à maints égards passionnant, de refondation de la méthode de l'école de la science sociale. En bref, en vertu du contexte historique dans lequel il a vécu et en vertu aussi de son éloignement par rapport aux centres où se discutaient les principes de la science sociale, Léon Gérin a produit malgré lui une théorie et une méthode qui s’inscrivent parfaitement dans le développement d'une tradition sociologique nationale (et qui sont tout à la fois, empressons-nous d'ajouter, une contribution à la connaissance sociologique générale, l'un n’interdisant aucunement l'autre). Il faudrait répéter cette conclusion à analyser dans cette perspective les trois tomes de L'Introduction à la sociologie générale (Rocher, 1969) de Guy Rocher ou Le Lieu de l’homme (Dumont, 1968) de Fernand Dumont, l'un et l'autre ouvrages ignorant superbement les réalités locales tout en œuvrant à la constitution d'un espace sociologique qui tend à universaliser la singularité du parcours historique du Québec.

On s'étonnera peut-être de ne pas compter parmi les dimensions de constitution d'un champ disciplinaire national le développement institutionnel des établissements d'enseignement supérieur. Il est raisonnable de postuler que, pour un pays, ou une région, donné, le nombre élevé de professeurs et de chercheurs peut renforcer le sentiment de faire communauté. Nous avons noté au passage plus haut comment l'explosion du système universitaire dans les années 1960 a contribué à la vigueur d'un champ disciplinaire national. On ne saurait nier que le nombre peut souvent assurer la force d'un groupe. Il est pourtant évident, comme cela l'a été, par exemple, pour la France au moment de la fondation de l'école durkheimienne sous l'impulsion d'une poignée d'auteurs, que le simple nombre ne saurait en aucun cas être une mesure nécessaire ou décisive de l'inscription d'un champ national. Ainsi, au moment de l'essor sans précédent des universités canadiennes, dans les années 1960, ce sont les théories et les méthodes de la sociologie américaine qui dominent au Canada anglais. Les professeurs de l'Université de Toronto ou de l'Université Carleton (dont les programmes de troisième cycle sont les mieux établis) sont directement branchés sur les revues et les associations de sociologie américaines. Par opposition, le faible niveau d'institutionnalisation dans l'enseignement supérieur de la sociologie doctrinale ${ }^{15}$ des années 1930

15. Nous appelons sociologie doctrinale la sociologie pratiquée par les sociologues catholiques québécois de l'entre-deux-guerres (Warren, 2003a). 
n'a pas empêché une fermeture face aux courants de pensée sociologique états-uniens, fermeture justifiée par l'ambition de fonder une pratique scientifique en rupture avec le matérialisme et le libéralisme supposés de la science protestante des grands voisins du Sud. On trouvera un autre exemple de cette faible correspondance entre masse professorale et autonomie disciplinaire dans l'évolution du nombre de membres des associations professionnelles de sociologie au Canada. Alors que les années 1970 et 1980 avaient correspondu à la création de trois associations régionales (Atlantic Association of Sociology and Anthropology, Western Association of Sociology and Anthropology et Ontario Sociological Association), la croissance des départements dans toutes les provinces canadiennes (même faible) n’a pas empêché le déclin, puis la disparition de ces trois associations dans les années 1990. L'identité régionale n'était pas assez forte pour maintenir l'existence d'un regroupement distinct, même si, en Ontario, les professeurs et professionnels de sociologie sont plus nombreux qu'au Québec, où l'Association de sociologie et d'anthropologie de langue française continue, bon an mal an, d'exister (Warren, 2004a). Cette remarque vaut pour l'Association canadiennefrançaise pour l'avancement des sciences (ACFAS), une association créée sur le modèle de ce qui se faisait aux États-Unis et en Angleterre et qui n'a pourtant pas son équivalent canadien-anglais, ayant été fondée en 1923, à un moment où l'identité collective canadienne-anglaise peinait à se libérer de sa référence impériale.

Il est tout aussi raisonnable de penser que les divergences idéologiques favorisent l'étanchéité des frontières nationales ${ }^{16}$; et vice versa, d'idées et d'idéaux semblables, suppose-t-on, ne peuvent vraisemblablement surgir des traditions disciplinaires distinctes. L'histoire de la sociologie franco-québécoise nous apprend pourtant le contraire. Il suffit de comparer l'évolution de sa traditionalisation avec celle de la sociologie canadienne anglophone pour être frappé par le peu de dialogue entre deux champs disciplinaires habités, tout au long du dernier siècle, par des idéologies très parentes. Si l'on fait exception de Léon Gérin, dont la méthode monographique n'avait pas d'égale dans le reste du Canada à la fin du XIX ${ }^{\mathrm{e}}$ siècle, il semble que le cliché des deux solitudes, comme nous l'avons mentionné plus haut, se répète une fois de plus dans l'histoire de la science sociale. Dans les années 1930, la sociologie doctrinale était proche, par ses idéaux et ses principes, de la sociologie issue de l'idéalisme écossais pratiquée à l'Université de Toronto par McIver ou Urwick (McKillop, 1994); seulement, l'humanisme thomiste dont elle faisait la promotion sur le plan intellectuel et le corporatisme dont elle prenait la défense sur le plan politique ne pouvaient que susciter des malentendus au Canada anglais, sans compter son nationalisme ethnique des plus intolérants (Warren, 2004c). Dans les années 1940, l'école de Chicago aurait pu rapprocher les deux mondes académiques, anglophones et francophones, et c'est d'ailleurs à cette

16. C'est ainsi que Harry H. Hiller, après avoir évoqué, comme conditions nécessaires à l'émergence de sociologies nationales, des développements nationaux distincts, mentionne des idéologies dominantes divergentes et des différences dans l'institutionnalisation de la discipline (Hiller, 1979a). Si nous acceptons la première condition, nous refusons d'attribuer aux deux autres le poids que Hiller leur donne. 
époque que les collaborations sont les plus nombreuses, à un moment où une pensée fédéraliste commençait à s'articuler au Québec (Warren, 2003a). Curieusement, toutefois, c'est moins par le détour du Canada anglais (y compris l'Université McGill où enseignait Carl A. Dawson) que par l'entremise d'un Américain, Everett C. Hughes, que cette réconciliation s'est faite. Autre manière de dire que l'ouverture fut d'abord une ouverture à l'Amérique plutôt qu'une ouverture au reste du Canada. Dans les années 1950, le fonctionnalisme gagnait en influence sur le continent et promettait l'établissement d'une science du social objective et universelle. Un des rares sociologues à avoir fait pendant longtemps le pont entre le Canada anglais et le Canada français, Guy Rocher n'eut que de minces collaborations avec ses collègues de Toronto ou Vancouver. Dans les années 1960, la pensée de la décolonisation, au lieu de rapprocher les deux solitudes en les soudant à un idéal révolutionnaire commun, les a éloignées l'une de l'autre, dans la mesure où si, pour les Canadiens anglais, la menace découlait des volontés de domination de l'impérialisme américain, pour les Québécois francophones, elle venait d'abord de l'impérialisme canadien anglais (Warren, 2003c; 2004b). La théorie de la dépendance, commune à plusieurs sociologues influencés par André Gunder Frank et consorts, servit ainsi, quelques belles exceptions mises à part, à isoler les deux communautés sociologiques. Dans les années 1970, le marxisme florissant ne permit pas davantage de lier les destinées des traditions canadienne-anglaise et franco-québécoise. En ces années, la vaste majorité des travaux publiés par les marxistes franco-québécois (d'obédiences diverses, sauf maoïste) tourne autour de la question nationale. «Nous croyons, écrivait Gilles Bourque et Nicole Laurin, que la nécessité et l'urgence pour le mouvement révolutionnaire d'orienter clairement et conséquemment sa pratique en fonction de ce qu'il est convenu d'appeler la question nationale exigent que l'on fasse l'effort de dégager au moins les éléments de base d'une théorie marxiste de la nation, du nationalisme et des rapports entre classes sociales, nations et idéologies nationalistes» (Bourque et Laurin, 1971). Or, il ne faisait aucun doute pour ces auteurs que la nation en question, c'était la nation québécoise, dont ils définissaient les contours avec autant de flous qu'ils mettaient de froide méticulosité à définir les lignes de démarcation d'une série plus ou moins hiérarchisée de classes sociales (Warren, 2004b). Dans les années 1980, l'essor des groupes de recherche, avec subséquemment la quête effrénée de réseaux, aurait pu contribuer à un rapprochement entre l'une et l'autre traditions, mais Paris, Berlin ou Boston ont toujours paru plus attirants que Vancouver, Saint John ou Edmonton. Enfin, dans les années 1990, la popularité croissante du thème de la mondialisation aurait pu aussi permettre de fructueux échanges à un moment où était célébrée ou décriée l'abolition des frontières nationales (avec, entre autres, la marginalisation des États nationaux), mais, encore une fois, dans leur volonté d'embrasser le monde entier, les sociologues franco-québécois ont ignoré ce qui se faisait de l'autre côté de l'Outaouais.

Le résultat de ce dédoublement de la sociologie canadienne fut d'isoler presque entièrement les deux traditions. Les grands noms de la discipline, John Porter, Fernand Dumont, Michel Freitag, attendent toujours leur traduction dans l'autre langue. Les 
auteurs canadiens de référence sont aussi étrangers qu'il se peut entre les sociologues de Colombie-Britannique et leurs collègues du Québec francophone.

Il vient d'être dit que la convergence des idéologies n'assure pas l'établissement d'un seul champ disciplinaire; la réciproque est aussi vraie: les divergences idéologiques ne provoquent pas nécessairement l'éclatement d'un champ en plusieurs champs autonomes. Il existait bien telle chose qu'un champ sociologique franco-québécois dans les années 1960, mais cela ne veut absolument pas dire que ce dernier était homogène, loin de là. L'on y retrouve comme ailleurs, malgré sa taille institutionnelle relativement réduite, des partisans qui du structuralisme, qui du marxisme, qui de l'herméneutique, qui de l'École de Chicago, etc. Ce qui fait se rejoindre Guy Rocher, Gilles Bourque et Fernand Dumont, c'est la reconnaissance d'un horizon national sur lequel se découpent la recherche et l'analyse scientifiques, l'idée de faire partie d'une histoire commune de la connaissance et le partage d'une certaine conception de la science - assez floue, doit-on le dire, ce qui explique, encore une fois, par comparaison avec ailleurs (les États-Unis et la France surtout), la faible traditionalisation du champ disciplinaire du Québec francophone.

À noter qu'au Canada anglais, les dimensions ont été isomorphes, et pour une période synchrone. Après un champ disciplinaire structuré à moitié par l'idéalisme britannique (MacIver et Urwick) et à moitié par l'écologie sociale de Chicago (Dawson), la sociologie canadienne de langue anglaise, malgré de vives et nombreuses oppositions $^{17}$, a voulu délimiter les frontières d'une société nationale globale. Le succès du livre de John Porter, The Vertical Mosaic, tient entre autres au fait que, pour la première fois avec une telle envergure, la société canadienne était considérée comme un tout, avec ses relations de pouvoir, sa stratification particulière, sa morphologie propre ${ }^{18}$. Il ne fut pas longtemps avant que des articles soient écrits et que des conférences soient données sur la tradition sociologique anglophone. Harold Innis, Samuel D. Clark, Carl A. Dawson et Leonard Marsh reçurent le statut de pères fondateurs. On fut pressé d'établir que la réflexion critique sur la société canadienne n'empruntait pas entièrement ses matériaux à l'étranger, mais qu'il existait une filiation intellectuelle qui remontait loin dans le temps et qui assurait aux auteurs contemporains une sorte de caution intellectuelle, voire morale. Les praticiens de la théorie de la dépendance furent, et pour cause, parmi les premiers à souligner le caractère indigène de leurs études et de leurs analyses (Drache, 1978). Ce mouvement culmina, dans les années 1980, en même temps que pour la sociologie canadienne de langue française, avec la publication de quelques ouvrages importants qui récapitulaient l'histoire de la discipline en même temps qu'ils

17. Par exemple, Bernard Gustin, en réaction à la controverse suscitée par les positions de Mathews et Steele (1969), déclarait: "Unlike folk songs and apple strudel, knowledge has no national character [...]. There is no such thing as Canadian sociology as distinct from Cuban sociology as distinct from American sociology» (cité par Mathews et Steele, 1969, p. 124-125).

18. Il faut être d'accord avec Rodney Crook lorsque ce dernier affirme que «the problem of a distinct English-Canadian sociology is precisely an expression of the problem of Canadian nationhood itself» (Crook, 1975). 
la légitimaient, en montrant qu'elle était constitutive du développement de la nation ${ }^{19}$. Enfin, on voulut montrer que les méthodes et les théories adoptées par les sociologues canadiens-anglais étaient originales en Amérique du Nord et qu'elles s'écartaient donc du modèle mis de l'avant par les sociologues américains. Un de ceux qui a le plus fait pour défendre une conception indigène de la sociologie canadienne-anglaise, Harry Hiller, pouvait affirmer que celle-ci se caractérisait par une approche plus globale qui lui faisait prendre pour objet la société canadienne comme un tout, par une relation ambivalente à la sociologie quantitative américaine et par la popularité de l'économie politique comme paradigme interprétatif (Hiller, 1990). Il aurait pu ajouter, avec plusieurs autres auteurs, dont Wallace Clement, qu'elle était aussi plus historique et plus critique que la sociologie pratiquée au sud du $45^{\mathrm{e}}$ parallèle (Clement, 1977; Drache, 1978).

Si le grand débat de la sociologie franco-québécoise des années 1970 fut celui de l'indépendance nationale, le grand débat de ces années au Canada anglais fut celui de la canadianisation (Canadianization debate) (Cormier, 2004). Ce débat était exacerbé par la domination numérique et académique des citoyens et des diplômés étrangers, en particulier américains, dans les départements de sociologie au pays. Dans un premier temps, les discussions ont tourné autour des possibilités d'embauche pour les docteurs canadiens, alors que leur nombre ne cessait de croître (35 maîtrises et 2 doctorats ont été décernés au Canada en 1960-1961, et 454 et 31, respectivement, dix ans plus tard) et que les perspectives d'avenir semblaient bouchées par la mainmise des professeurs étrangers, surtout américains, sur les universités canadiennes. Pour l'année universitaire 1970-1971, par exemple, $39 \%$ de tous les sociologues et anthropologues au Canada étaient citoyens américains, contre $40 \%$ qui étaient citoyens canadiens ${ }^{20}$. Dans certains départements de sociologie, en Alberta et en Colombie-Britannique, entre autres, la proportion de professeurs étrangers atteignait plus de $70 \%$. Dans un deuxième temps, les discussions ont traité, comme nous l'avons mentionné plus haut, de la pauvreté des études empiriques entreprises sur la réalité canadienne - les objets d'étude ne reflétaient guère ni les préoccupations ni les réalités sociales des Canadiens. Dans un troisième temps, le débat s'est déplacé vers une discussion des principes qui devaient guider l'enseignement de la sociologie et la recherche en ce domaine au Canada. Ce n'était plus alors tant les perspectives d'embauche ou les données concrètes que les bases épistémologiques sur lesquelles devait être établie la science sociologique qui devenaient l'objet de vives polémiques. Contre un certain nombre de sociologues américains enseignant dans des universités canadiennes, pour la plupart formés au courant fonctionnaliste, qui prétendaient à une science objective et universelle, plusieurs sociologues canadiens et américains (ces derniers étant venus s'établir au Canada en réaction à la politique conservatrice et impérialiste américaine) affirmaient que l'opposition

19. Citons, entre autres, Brym (1989), Hiller (1982a) et Shore (1986). Notons aussi, outre la préparation de deux numéros spéciaux de revue dans les années 1980, la publication de nombreux manuels et collectifs centrés autour de l'analyse de problèmes sociaux et réalités sociales propres à la société canadienne.

20. Tous les chiffres cités ici sont tirés de Hiller (1979). 
entre le centre et sa périphérie (Shils, 1961), c'est-à-dire entre l'empire et ses colonies, fondait des différences sur le plan des hypothèses et des perspectives de recherche. Il s'agissait donc, en réaction, comme cela se discutait ailleurs (en Scandinavie, en Australie, etc. [Genov, 1989]), d'établir une science indigène ( a nationalistic social social») en lieu et place d'une science importée et colonisatrice (Cormier, 2002; Rush, 1981).

Les trois dimensions dont il a été fait mention sont suffisantes (mais non nécessaires, dans la mesure où la reconnaissance du champ est un phénomène en partie rétrospectif) pour qu'un champ sociologique national soit reconnu comme tel. Il semble toutefois possible de concevoir plusieurs situations où un champ sociologique national existe sans que les praticiens qui gravitent au sein de celui-ci n'aient songé à replacer leurs objets d'étude dans l'horizon de la société globale, n'aient pensé à écrire une histoire nationale de leur discipline ou n'aient eu l'intention de développer des théories et des méthodes qui leur soient propres. Ce serait néanmoins par un abus de langage. L'Europe peut bien avoir constitué un champ sociologique distinct de celui des États-Unis dans les années 1930, ou l'École de Chicago (Chapoulie, 2001) avoir institué une pratique distincte de celle de l'Université Columbia dans les mêmes années, il faudrait faire attention à ne pas trahir les termes dont l'historien des sciences se sert pour délimiter des aires de traditionalisation. Un champ sociologique national est finalement aussi flou et aussi fragile que la nation elle-même. C'est ce qui explique que les critères utilisés pour le définir recoupent ceux dont on se sert d'ordinaire pour définir la nation (une référence collective, une histoire, des valeurs censément propres [Dumont, 1995]) et que les moments de poussée nationaliste, comme au Québec dans les années 1960 et 1970, sont aussi ceux où le processus de traditionalisation est le plus actif et puissant. Il n'en demeure pas moins que, dans l'histoire générale de la sociologie, de telles aires ont été constituées et ont su conserver une consistance assez grande pour que l'on puisse parler, avec à l'évidence toutes les nuances qui s'imposent, d'une tradition française, d'une tradition britannique, d'une tradition italienne, d'une tradition américaine ou d'une tradition franco-québécoise.

\section{UNIVERSALISATION, INTERNATIONALISATION OU MONDIALISATION ?}

Nous en sommes resté tout au long de cet article à un niveau très théorique. C’est que la mesure concrète de l'universalisation d'une discipline est un exercice qui, sans être impossible, est laborieux et contestable. L'évaluation de la clôture du champ institutionnel peut être tentée à partir de critères relativement clairs et objectifs, des critères qui se laissent quantifier: la circulation des personnes et des textes ou le financement de la recherche, par exemple. La clôture du champ «scientifique» est, quant à elle, beaucoup plus difficile à saisir. Quand même on pourrait mettre un chiffre approximatif sur l'influence française et américaine sur le développement de la sociologie franco-québécoise, on ne pourrait savoir encore dans quelle mesure la combinaison de ces deux influences a pu donner lieu à une tradition originale. Il n'en est pas moins intéressant de se demander en conclusion si les champs sociologiques nationaux ont encore un ave- 
nir à l'heure de la mondialisation et de la retraduction de leur ancienne ambition de synthèse en pures visées instrumentales (Genov, 1989b). Il est certain, en première analyse, que l'on assiste à une plus grande intégration, sur le plan formel et institutionnel, des traditions sociologiques nationales depuis quelques années, et ce dans le mouvement même où les anciens repères identitaires collectifs (l’État-nation, en particulier) sont remis en question.

Selon une première hypothèse, on pourrait prétendre que cette internationalisation s'inscrit dans un processus normal de professionnalisation (Beaver et Rosen, 1979) (dont un des indices est l'augmentation des subventions et le développement des instances organisationnelles) et que, par conséquent, la dénationalisation de la discipline sociologique est un phénomène aussi prévisible qu'inéluctable (Crawford, Shinn et Sörlin, 1984). «[...] the sciences are certainly still being nationalized — but [...] denationalizing science is to us the prevailing trend» (Crawford, Shinn et Sörlin, 1993). Malgré le fait que la pratique sociologique, de par sa nature propre, continue à lui offrir une plus grande résistance que les sciences dites exactes, les chiffres compilés par les chercheurs tendent à démontrer l'internationalisation de la pratique scientifique ${ }^{21}$. Il faudrait, soutiennent certains, se réjouir de cette tendance de fond qui viendrait non seulement couronner les efforts de reconnaissance des chercheurs locaux mais accréditer l'idée que la sociologie, en retard sur l'essor historique des autres disciplines académiques, est en voie de s'établir comme science véritable, donc universelle.

Throughout the 1970s, 1980s and 1990s, Anglo-Canadian sociology was busy chasing an illusionary «holy grail» of a distinctive Canadian sociology. [...] Canada's close but ambivalent relationship to the United States allows us to draw from the strengths of American sociology, while moving beyond some of its blindness and parochialisms. (McLaughlin, 2005, p. 30)

Le genre de nationalisme mis de l'avant dans les années 1970 et 1980 aurait légitimé "precisely the kind of anti-professionalism, self-interested careerism and radical posturing that has done such harm to Canadian sociology over the past decades " (McLaughlin, 2004, p. 95). Pour accomplir un saut hors du provincialisme, McLaughlin suggère donc d'accentuer le caractère professionnel de la discipline en relevant les critères d'excellence en recherche, en publication et en enseignement. Alors seulement y aura-t-il une science sociologique possible ( «a possible sociological science») parce que cette sociologie se sera faite, justement, science.

Selon une deuxième ligne d'interprétation, il faudrait distinguer davantage universalisation et mondialisation, celle-ci s'accompagnant d'un affaiblissement des frontières nationales - ce qui expliquerait pourquoi, entre autres, l'économie, la gestion et

21. En 1995, les publications canadiennes en sciences sociales et humaines écrites en collaboration internationale atteignaient environ $18 \%$, en hausse de plus de $50 \%$ depuis 1981 (sauf pour Terre-Neuve et le Nouveau-Brunswick, dont le nombre de collaborations est trop faible, les différences entre les provinces sont négligeables). Les montants de contrats et subventions d'origine étrangère obtenus par les chercheurs universitaires canadiens sont aussi nettement à la hausse (Lebel et Larivière, 2004; Gingras, Godin et Foisy, 1999). 
le management sont les plus internationales des science sociales, puisqu'elles sont associées de près à la globalisation (sans compter, il va sans dire, qu'elles se prêtent davantage à une formalisation, sinon à une mathématisation, du rapport à leur objet - ceci renforçant cela, et vice versa). Ce serait donc moins ici un effet du développement de la science sociologique, et donc de sa maturité, qui favorisait son intégration planétaire, qu'un simple enrôlement — inconscient ou volontaire — des sociologues dans le vaste processus de financiarisation globale du capital et de création d'un marché sans frontières (Newson, 1988; Slaughter et Leslie, 1999). Cette hypothèse trouvera plusieurs confirmations dans les pages de notre texte puisque, comme nous l'avons vu, c'est à l'intérieur de la dynamique de l'État-nation que les sociologues québécois ont œuvré à la constitution d'une tradition originale. C'est, par exemple, au moment où le mouvement néonationaliste emporte, dans les années 1970, une large partie de l'opinion provinciale et qu'est fondé le Parti québécois que les sociologues, intéressés quelques années plus tôt par des questions en apparence plus désincarnées, se mettent à creuser davantage la question nationale (Dumont, 1971; Rioux, 1969; Rocher, 1973).

De manière plus radicale, d'autres auteurs affirment que la tendance opérationnaliste de la sociotechnologie contemporaine tend à dissoudre la sociologie classique pour la remplacer par une pratique apatride; un savoir qui n'est plus qu'une technique vouée à l'amélioration des systèmes selon des critères de performance et d'efficience n'a que faire de la question de la société globale: tout pour elle se dissout en environnements dont les conditions écologiques doivent être contrôlées ou auxquels les individus doivent s'adapter de manière pragmatique, utilitaire, profitable. Aussi la société n'existerait plus, à peine existerait-il un système social, car tout se ramènerait à des objets de plus en plus manipulables et programmables (Freitag, 2002). L'histoire de la discipline serait évacuée au profit d'une description des modalités de la sociotechnologie, ce que traduit bien, dans les universités, la mode des manuels qui hachurent et découpent le réel en une série de tranches et de domaines. Il ne s'agirait plus d'abolir l'éventail des théories afin de les remplacer par une seule, la suprême théorie, qui serait en quelque sorte l'accomplissement de la promesse comtienne de science universelle, mais plutôt de les dissoudre en tant que théories et de les faire renaitre comme paradigmes. Dans ce dernier cas, les champs nationaux seraient réconciliés, non parce qu'y circulerait une théorie générale acceptée de tous, mais simplement parce que la question de l'utilisation d'une théorie ou d'une autre serait abolie et remplacée par le seul critère de la performance.

Pour notre part, nous ne croyons pas en être arrivé au stade où les champs sociologiques nationaux seraient sur le point de disparaître. Il demeure que leur existence importe aux praticiens des sciences sociales dans la mesure où ils permettent le maintien d'une question sur l'orientation et la nature de la pratique sociologique elle-même. Car, rappelons-le à ceux qui imaginent les champs nationaux comme des obstacles à l'établissement d'une science enfin universelle, comme autrefois les impérialistes voulaient faire des cultures indigènes des obstacles sur la voie de la civilisation, les champs disciplinaires nationaux ne sont rien de tels: dans la perspective de la mondialisation, 
ils sont des leviers; dans la perspective de la transformation sur un mode technocratique de nos disciplines, ils sont des questions.

Aujourd'hui encore, la tradition sociologique du Québec francophone demeure un levier particulièrement intéressant pour explorer les enjeux suscités par le nouvel ordre mondial, et une question particulièrement passionnante pour comprendre les défis posés par la transition postmoderne, en science comme en maints autres domaines.

\section{RÉSUMÉ}

Cet article s'intéresse à la fondation historique d'un champ disciplinaire national, c'est-à-dire au processus de traditionalisation scientifique. Plus spécifiquement, il cherche à mieux comprendre, et ce en la comparant sans cesse au développement de la sociologie du Canada anglophone, comment la sociologie du Québec francophone a progressivement créé un espace référentiel, méthodologique, théorique et politique, et comment celle-ci a cherché, surtout dans les années 1960 et 1970, à promouvoir une connaissance enracinée dans la réalité nationale. C'est ainsi que l'auteur est amené à souligner trois dimensions inhérentes à la constitution d'un champ sociologique national, soit la définition d'une société globale, l'écriture d'une histoire, ainsi que la formulation de méthodes et de théories spécifiques — marginalisant donc les dimensions idéologiques et institutionnelles invoquées par Harry H. Hiller dans ses travaux sur l'établissement des traditions sociologiques canadiennes.

\section{ABSTRACT}

This paper examines the historical foundation of a national disciplinary field, namely the process of scientific traditionalization. More specifically, it seeks a better understanding, through a continuous comparison with the development of sociology in English-speaking Canada, of how the sociology of French-speaking Quebec has gradually created a refential, methodological, theoretical and political space, and how it tried, especially in the ' 60 s and '70s, to promote knowledge rooted in the national reality. The author is thus led to emphasize three dimensions inherent in the constitution of a national sociological field, namely the definition of a global society, the writing of a history, as well as the formulation of specific methods and theories - thus marginalising the ideological and institutional dimensions invoked by Harry $\mathrm{H}$. Hiller in his studies on the establishment of Canadian sociological traditions.

\section{RESUMEN}

Este artículo se interesa por la fundación histórica de un campo disciplinario nacional, es decir, el proceso de tradicionalización científico. Más específicamente, pretende comprender mejor, y esto comparándolo sin cesar al desarrollo de la sociología del Canadá anglófono, cómo la sociología del Quebec francófono ha creado progresivamente un espacio de referencia, metodológico, teórico y político, y cómo ésta ha pretendido, sobre todo en los años 1960 y 1970, promover un conocimiento arraigado en la realidad nacional. Por ello el autor destaca tres dimensiones inherentes a la constitución de un campo sociológico nacional, o sea la definición de una sociedad global, la escritura de una historia, así como la formulación de métodos y teorías específicas - marginalizando entonces las dimensiones ideológicas e institucionales invocadas por Harry $\mathrm{H}$. Hiller en sus trabajos sobre el establecimiento de las tradiciones sociológicas canadienses-. 


\section{BIBLIOGRAPHIE}

Аввотт, A. (2001), Chaos of Disciplines, Chicago, University of Chicago Press.

BeAver, D. de B. ET R. Rosen (1979), «Studies in Scientific Collaboration», $1^{\text {re }}$ et $2^{\mathrm{e}}$ partie, Scientometrics, vol. $1, \mathrm{n}^{\circ} 1$ et $\mathrm{n}^{\circ} 3,1979$, p. 65-84 et p. 231-245.

Bourdieu, P. (2002a), «Quelques propriétés des champs», dans P. Bourdieu, Questions de sociologie, Paris, Éd. de Minuit, p. 113-120.

Bourdieu, P. (2002b), «Les conditions sociales de la circulation internationale des idées », Actes de la recherche en sciences sociales, 145, décembre, p. 3-8.

Bourdieu, P. (2000), «Deux universalismes en conflit», The Tocqueville Review, 21, 1, p.

Bouridue, P. (1992), «Deux impérialismes de l'universel», dans C. Fauré et T. Bishop, L'Amérique des Français, Paris, F. Bourin.

Bourdieu, P. (1976), «Le champ scientifique», Actes de la recherche en sciences sociales, vol. 3, p. 88-104.

Bourque, G. et N. Laurin (1971), «Classes sociales et idéologies nationalistes au Québec», Socialisme québécois, 21-22, p. 109-155.

Brooks, S. et A.-G. GAGnon (1994), Les spécialistes des sciences sociales et la politique au Canada. Entre l'ordre des clercs et l'avant-garde, Montréal, Boréal.

Brym, R. et B. Fox (1989), From Culture to Power: The Sociology of English Canada, Toronto, Oxford University Press.

Chapoulie, J.-M. (2001), La tradition sociologique de Chicago (1892-1961), Paris, Le Seuil.

Clement, W. (1977), «Macrosociological Approaches Toward a 'Canadian Sociology'», Alternate Route, vol. 1, p. 1-37.

Coburn, D. (1970), "Sociology and Sociologists in Canada: Problems and Prospects», in Jan J. Loubser (dir.), The Future of Sociology in Canada/L'avenir de la sociologie au Canada, Montréal, Société canadienne de sociologie et d'anthropologie, p. 36-59.

Cormier, J. (2004), The Canadianization Movement. Emergence, Survival, and Success, Toronto, University of Toronto Press.

Cormier, J. (2002), «Nationalism, Activism, and Canadian Sociology», The American Sociologist, vol. 33 (1), p. $12-41$.

Crane, D. (1972), Invisible Colleges. Diffusion of Knowledge in Scientific Communities, Chicago et Londres, The University of Chicago Press.

Crawford E., T. Shinn et S. Sörlin (1993), Denationalizing Science. The Contexts of International Scientific Practice, Dordretch, Kluwer Academic.

Crook, R. (1975), «Teaching and Learning Sociology», in D. Forcese et S. Richer (dir.), Issues in Canadian Society: an Introduction to Sociology, Scarborough, Prentice-Hall, p. 467-498.

Davis, A. K. (1971), «Canadian Society and History as Hinterland Versus Metropolis», dans: R. J. Ossenberg (dir.), Canadian Society: Pluralism, Change, and Conflict, Scarborough, Prentice-Hall, p. 6-32.

Dion, S. (1991), «Le nationalisme dans la convergence culturelle. Le Québec contemporain et le paradoxe de Tocqueville», dans R. Hudon et R. Pelletier (dir.), L'engagement intellectuel: mélanges en l'honneur de Léon Dion, Sainte-Foy, Les Presses de l'Université Laval, 1991, p. 291-311.

Drache, D. (1978), «Rediscovering Canadian Political Economy», dans W. Clement et D. Drache, A Practical Guide to Canadian Political Economy, James Lorimier, Toronto, p. 1-53.

Dumont, F. (1995), Raisons communes, Montréal, Boréal.

Dumont, F. (1993), Genèse de la société québécoise, Montréal, Boréal.

Dumont, F. (1976), «Le projet d’une histoire de la pensée québécoise», dans Philosophie au Québec, Montréal, Bellarmin-Desclée, p. 23-48.

Dumont, F. (1974), Les idéologies, Paris, PUF.

Dumont, F. (1971), Vigile du Québec, Montréal, HMH.

Dumont, Fernand (1968), Le Lieu de l'homme. La culture comme distance et mémoire, Montréal, Éditions $\mathrm{HMH}$. 
Dumont, F. (1962), "L'étude systématique de la société globale canadienne-française», Recherches sociographiques, vol. III, $\mathrm{n}^{\circ}$ 1-2, janvier-août, p. 277-294.

Falardeau, J.-C. (1953), Essais sur le Québec contemporain/Essays on Contemporary Quebec, Québec, Presses de l'Université Laval.

Falardeau, J.-C. et F. Jones (1956), «La sociologie au Canada », Actes du Troisième congrès mondial de sociologie, vol. VII, La sociologie en 1956, Amsterdam, Association internationale de sociologie, p. 14-22.

Fournier, M. (2002), «Quebec Sociology: A discipline and its Objet», The American Sociologist, 33, 1, p. $42-54$.

Fournier, M. (2001), "Quebec Sociology and Quebec Society: The Construction of a Collective Identity», Canadian Journal of Sociology, vol. XXVI, $\mathrm{n}^{\circ}$ 3, p. 333-347.

Fournier, M. (2000), «Notes pour une histoire de la sociologie québécoise suivies d'éléments de réflexion sur l'avenir», dans Robert Lahaise (dir.), Québec 2000, Montréal, HMH, p. 287-322

Fournier, M. (1986), L'Entrée dans la modernité, Montréal, Éditions Saint-Martin.

Fournier, M., A. Germain, Y. Lamarche et L. Maheu (1975), «Le champ scientifique québécois: structure, fonctionnement et fonctions", Sociologie et sociétés, vol. 7, $\mathrm{n}^{\circ}$ 1, mai, p. 119-132.

Freitag, M. (2002), L'oubli de la société, Pour une théorie critique de la postmodernité, Québec, PUL.

GAGNÉ, G. et J.-P. WARren (2003), «La sociologie québécoise d'expression française: du fait de la morale à la morale du fait», dans G. Gagné et J.-P. Warren (dir.), Sociologie et valeurs. Quatorze penseurs québécois $d u X X^{e}$ siècle, Montréal, Les Presses Universitaires de Montréal, p. 7-44.

Garretôn, M. A., M. Murmis, G. De Sierra et H. Trindade(2005), «Social Sciences in Latin America: a Comparative Perspective - Argentina, Brazil, Chile, Mexico and Uruguay», Information sur les sciences sociales, vol. 44, n 2-3, p. 557-593.

Genov, N. (dir.) (1989a), National Traditions in Sociology, Londres, Sage.

Genov, N. (1989a), "National Sociological Traditions and the Internationalization of Sociology», dans N. Genov (dir.), National Traditions in Sociology, Londres, Sage.

Gingras, Y. (2003), «Mathématisation et exclusion: socio-analyse de la formation des cités savantes», dans Jean-Jacques Wunenburger, Bachelard et l'épistémologie française, Paris, PUF, p. 115-152.

Gingras, Y. (2002), «Les formes spécifiques de l'internationalité du champ scientifique», Actes de la recherche en sciences sociales, 141-142, mars, p. 31-45.

Gingras, Y., B. Godin et M. Foisy (1999), «L'internationalisation de la recherche universitaire au Canada», dans S. Bond et J.-P. Lemasson, Un nouveau monde du savoir. Les universités canadiennes et la mondialisation, Ottawa, Centre de recherches pour le développement international, p. 77-98.

Guppy, N. et B. Arai (1994), «Teaching Sociology: Comparing Undergraduate Curricula in the United States and in English Canada», Teaching Sociology, 22, 3, p. 217-240.

Hartsock, N. (1999), "Mary O'Brien's Contributions to Contemporary Feminist Theory», Les Cahiers de la femme, vol. $18, \mathrm{n}^{\circ} 4$, hiver.

Hiller, H. (1990), «Anglophone Canadian Sociology: Context and Evolution», in R. Breton et al. (dir.), National Survival in Dependent Societies. Social Change in Canada and Poland, Ottawa, Carleton University Press, p. 29-42.

Hiller, H. (1982a), Society and Change: S. D. Clarke and the Development of Canadian Sociology, Toronto, University of Toronto Press.

Hiller, H. (1982b), «Nationality, Relevance, and Ethnocentrism: An Essay in the Sociology of Canadian Book Publishing», Social Forces, 59, 4, p. 1297-1307.

Hiller, H. (1979a), "Universality of Science and the Question of National Sociologies», The American Sociologist, vol. 14, no 3, août, p. 124-135.

Hiller, H. (1979b), «The Canadian Sociology Movement: Analysis and Assessment», The Canadian Journal of Sociology, vol. 4, p. 125-150.

Hofley, J. R. (1992), «Canadianization: A Journey Completed?», in W. K. Carroll et al. (dir.), Fragile Truths. Twenty-Five Years of Sociology and Anthropology in Canada, Ottawa, Carleton University Press, p. 105. 
Hughes, E. C. (1977), Fonds d'archives Everett C. Hughes, Chicago, University of Chicago Library.

Hughes, E. C. (1943), Programmes de recherches sociales pour le Québec, Cahiers de l'École de Sciences sociales, politiques et économiques de l'Université Laval, 2, 4.

KazAncigit, A. et D. Makison (2001), Les sciences sociales dans le monde, Paris, UNESCO et Éditions de la Maison des sciences de l'homme.

Lebel, J. et V. LaRivière (2004), «Publications à auteurs multiples: croissance régulière des partenariats internationaux", L'observation Science et technologie, note n ${ }^{\circ} 4,12$ janvier, 7 pages.

LÉvesque, G.-H. et al. (dir.) (1984), Continuité et ruptures. Les sciences sociales au Québec, Québec, PUL.

Macpherson, C. B. (1957), «The Social Sciences», in Julian Park (dir.), The Culture of Contemporary Canada, Ithaca, Cornell University Press.

Mathews, R. et J. Steele (dir.) (1969), The Struggle for Canadian Universities, New Press.

McKillop, B. (1994), Matters of Mind: The University in Ontario, 1791-1951, Toronto, University of Toronto Press.

McLaughuin, Neil (2005), "Canada's Impossible Science: Historical and Institutional Origins of the Coming Crisis in Anglo-Canadian Sociology», Canadian Journal of Sociology, 30 (1), p. 1-40.

McLaughlin, Neil (2004), "A Canadian Rejoinder: Sociology North and South of the Border", The American Sociologist, vol. 35 (1), p. 80-101.

Morris, R. (1993), «Les conventions littéraires de l'écriture sociologiques au Québec et au Canada anglais», dans André Turmel (dir.), Chantiers sociologiques et anthropologiques, Montréal, Éditions du Méridien, p. 15-24.

Newson, J. (1988), «The Corporate-Linked University: From Social Project to Market Force», Canadian Journal of Communications, vol. $23, \mathrm{n}^{\circ} 1$.

Nielsen, G. et J.-P. Warren, «La sociologie canadienne anglophone: le défi d'une définition», Cahiers de recherche sociologique, 39, p. 5-14.

Nisbet, R. (1984), La tradition sociologique, Paris, PUF.

Rioux, M. (1969), La Question du Québec, Montréal, Parti pris, 1969.

Rocher, G. (1990), «Les deux solitudes chez les sociologues canadiens», Society/Société, 14, 2, p. 3-10.

Rocher, G. (1973), Le Québec en mutation, Montréal, HMH-Hurtubise.

Rocher, G. (1969), L'Introduction à la sociologie générale, Montréal, Éditions HMH.

Rush, G. B. (1981), «Lament for a Notion: the development of social science in Canada», Revue canadienne de sociologie et d'anthropologie, 18, 4, p. 519-544.

SALÉE, D. (1999), «Innis and Quebec: The Paradigm That Would not Be», dans C. R. Acland et W. J. Buxton (dir.), Harold Innis in the New Century, Montréal et Kingston, McGill-Queen's Press.

Schwartz, M. A. (1991), «Scientifiques et intellectuels au Canada francophone et au Canada anglophone», dans R. Hudon et R. Pelletier (dir.), L'engagement intellectuel. Mélanges en l'honneur de Léon Dion, Sainte-Foy, Les Presses de l'Université Laval, p. 403-415.

SHiLs, E. (1961), «Center and Periphery», dans The Logic of Personal Knowledge, Londres, Routledge, p. 117130.

Shore, M. (1987), The Science of Social Redemption. McGill, the Chicago School, and the Origins of Social Research in Canada, Toronto, The University of Toronto Press.

Slaughter, S. et L. Leslie (1999), Academic Capitalism: Politics, Policies and the Entrepreneurial University, Baltimore, Johns Hopkins University Press.

Soutнсотт, C. (1994), «The Study of Regionalism in Quebec and English Canada: a comparative analysis of perspective», Canadian Journal of Sociology, 25, 4, décembre, p. 457-484.

Stolzman, J. et H. Gamberg (1975), «The National Question and Canadian Sociology», Canadian Journal of Sociology, 1, 1, p. 91-106.

Trindade, H. (2005), «Les sciences sociales en Amérique latine (1930-2003): Présentation», Information sur les sciences sociales, vol. 44, $\mathrm{n}^{\circ}$ 2-3, p. 219-226.

WADE, M. (1960), Canadian Dualism: Studies of French-English Relations, Toronto et Québec, University of Toronto Press et Presses de l'Université Laval. 
WARREN, J.-P. (2005), «Internationalisation de la formation doctorale? Le cas des professeurs universitaires de sociologie», Cahier de l'ACSALF, vol. 2, n 2, avril, p. 15-16.

Warren, J.-P. (2004a), «La déroute des associations professionnelles de sociologie», Cahier de l'Acsalf, vol. 1, no 1 , avril, p. 19-21.

WARren, J.-P. (2004b), «Nation, révolution et indépendance. Considérations générales sur l'évolution de la sociologie critique au Canada français et au Canada anglais», Bulletin d'histoire politique, vol. 12, $\mathrm{n}^{\circ} 3$, printemps-été, p. 118-132.

WARREN, J.-P. (2004c), «Sciences sociales et religions chrétiennes au Canada», Revue d'histoire de l'Amérique française, vol. 57, $\mathrm{n}^{\circ} 3$, hiver, p. 407-424.

WARren, J.-P. (2003a), L'engagement sociologique. La tradition sociologique du Québec francophone, Montréal, Boréal.

WARren, J.-P. (avec la collaboration de G. Nielsen) (dir.) (2003b), La tradition sociologique canadienne anglophone, numéro spécial des Cahiers de recherche sociologique, 39.

Warren, J.-P. (2003c), «Le Canada: un produit des empires», Cahiers de recherche sociologique, 39, p. 6992. 